\title{
Adaptive Encoding Algorithm for Multispectral Images
}

\author{
S.Deepa \\ Research Scholar, \\ Department of Computer Science and Engineering, \\ Manonmaniam Sundaranar University, \\ Tirunelveli, Tamilnadu
}

\begin{abstract}
A new adaptive multispectral image compression technique based on the regions identified is proposed. The algorithm is adaptive in the sense that according to the data type class of the region, appropriate encoding technique is chosen. The image is first segmented by means of Region splitting and merging procedure based on the statistical characteristic of the image. Then class adaptive hotelling transform or Karhunen Loeve transform (KLT) in the spectral domain and the shape adaptive wavelet transform in the spatial domain are adopted in the image by considering the spatial, spectral and statistical properties which are unique to the multispectral images. The quadtree is used for determining the transform block size and a single KLT matrix is used for the regions of same class ie., class adaptive KLT is applied and the transformation is followed by shape adaptive wavelet transform(SAWT) incorporating the spatial and structural properties of the multispectral image. After transformation, based on the regions identified, if the region is relatively uniform or smooth, the SPIHT (Set Partitioning in Hierarchical Trees) algorithm is adopted. If not, that is, if the region is highly textured in nature, then object based wavelet method is used for compression. Thus the advantages of both SPIHT algorithm and object based wavelet encoding method, both in terms of visual quality and PSNR values, are incorporated in a single compression technique.
\end{abstract}

Key words: class adaptive KL transform, Quadtree, shape adaptive WT, Adaptive encoding technique

\section{INTRODUCTION}

Multispectral Image, as the name defines, an image which includes several bands in the visual and infrared regions of the electromagnetic spectrum captured by remote sensing radiometers. They deliver images with very high spatial, spectral and radiometric resolution. But transmitting such a huge amount of data to the ground station on limited-capacity channels, and to the end users on common transmission facilities deserves a high quality compression standard. Therefore, suitable compression algorithms play a central role for the success of remote sensing applications.

The existing standard compression algorithms, like the JPEG, JPEG2000, or MPEG standards, do not provide satisfactory results for multispectral data, because they do not focus on the statistical features. Thus, several ad hoc compression techniques have been proposed in the last few years. Earlier researches concentrate on using different types of transformation like DCT, KLT and WT etc. Later the researchers combined the transformation in spatial and spectral domain [9]. After trying out different combinations, researchers focused on the encoding algorithms [11,23,24]. The encoding algorithms may be based on predictive coding[25], vector quantization[1,11] or transform coding $[12,15]$. Most of them rely on transform coding because of its simplicity and good results[5].

One of the best compression technique for multispectral images designed so far is done on region based transform coding [24]. Here, the image is segmented (using Tree structured Markov random field model[22,23]) into a number of disjoint regions, based prevalently on their spectral features, which are then transform encoded. (Transformation by KLT and Wavelet Transform, Encoding by SPIHT). This technique shows good performance at medium bit-rates, showing compression, at an appropriate coding rate. The users are concerned about particular regions of interest like mountains, rivers etc. and thereby used in data mining applications. Hence, the performance is measured by computing the misclassification rate, that is, the fraction of erroneously labeled pixels with respect to a reference classification. The region-based coder provides often, although not always, the best results, confirming its performance advantage. Of course, the error is larger for the image, where less spectral information is available.

In the research work, the proposed image segmentation is to be based both on their statistical as well as spectral features[13,19] so that we can minimize the average loss in misclassification. Moreover, for better 
performance, the different regions of the image are coded by means of different encoding techniques, say, SPIHT algorithm for relatively uniform regions and modified Object Wavelet method for highly textured areas, which are quite common in remote-sensing images[20].

A generic transform coder involves transformation,

Image $\rightarrow$ Transform $\rightarrow$ Quantize $\rightarrow$ Encode $\rightarrow$ Compressed

FIG 1. GENERAL TRANSFORM CODER

quantization and encoding, as shown in Fig. 1. The original image is passed through some transformation to produce transform coefficients. The goal of the transformation is to produce coefficients that are decorrelated. It removes the spatial/spectral dependencies. The decorrelated coefficients can be encoded efficiently for compression. Quantization is done to remove the bits which are considered to be unwanted or redundant bits. This step is neglected if we perform a lossless compression. The last step, encoding, is the place where the compression algorithm is actually applied and the output is a compressed image.

In this paper, section II describes segmentation which plays a key role in the proposed work. Section III and IV explores the transformation in spectral and spatial domain respectively. The next section describes the proposed algorithm followed by conclusion and future work.

\section{SEGMENTATION BY REGION SPLITTING AND MERGING}

Classification is carried out by means of segmentation. Segmentation subdivides an image into its constituent regions or objects. The level of detail to which the subdivision is carried depends on the problem being solved[6]. That is, segmentation should stop when the objects or regions of interest in an application have been detected. To solve a simple segmentation problem is a predicate that differentiates between textured and constant regions. The standard deviation of pixel values is a measure that accomplishes this, because it is nonzero in areas of the texture region and zero otherwise.

Segmentation is achieved by attempting to find boundaries between regions based on discontinuities in intensity levels or via thresholds based on the distribution of pixel properties, such as intensity values or color. The segmentation techniques (a) Region growing and (b) Region splitting and merging are based on finding the regions directly. Region growing is a procedure that groups pixels or subregions into larger regions based on predefined criteria. The selection of criteria depends not only on the problem under consideration, but also on the type of image data available. For example, the analysis of Multispectral imagery depends heavily on the use of color. This problem would be significantly more difficult, or even impossible, to solve without the inherent information available in color images. Another problem in region growing is the formulation of a stopping rule. Region growth should stop when no more pixels satisfy the criteria for inclusion in that region. Criteria such as intensity values, texture, and color are local in nature and do not take into account the 'history' of region growth.

Thus the alternative technique 'Region splitting and merging' is chosen to segment the Multispectral image. The procedure is to subdivide an image initially into a set of arbitrary, disjoint regions and then merge and/or split the regions in an attempt to satisfy the conditions of segmentation stated below. Let $\mathrm{R}$ represent the entire spatial region occupied by the multispectral image. $\mathrm{R}$ is partitioned into $\mathrm{n}$ subregions, $\mathrm{R} 1, \mathrm{R} 2, \ldots, \mathrm{Rn}$, such that, for $\mathrm{i}=1,2, \ldots, \mathrm{n}$

(a) $U\left(R_{i}\right)=R$

(b) $\mathrm{R}_{\mathrm{i}}$ is a connected set

(c) $\mathrm{R}_{\mathrm{i}} \cap \mathrm{R}_{\mathrm{j}}=\varnothing$

(d) $\mathrm{Q}\left(\mathrm{R}_{\mathrm{i}}\right) \mathrm{TRUE}$

(e) $\mathrm{Q}\left(\mathrm{R}_{\mathrm{i}} \cup \mathrm{R}_{\mathrm{j}}\right)=\mathrm{FALSE}$ for any adjacent regions $\mathrm{R}_{\mathrm{i}}$ and $\mathrm{R}_{\mathrm{j}}$

Here, $Q\left(R_{i}\right)$ is a logical predicate defined over the points in set $R_{i}$ and $\varnothing$ is the null set. Two regions $R_{i}$ and $R_{j}$ are said to be adjacent if their union forms a connected set.

Let $\mathrm{R}$ represent the entire image region and a predicate [20] is selected. The predicate is based on the mean vector, covariance matrix etc.

$$
\mathrm{x} \varepsilon \mathrm{w}_{\mathrm{i}} \text { if } \mathrm{g}_{\mathrm{i}}(\mathrm{x})>\mathrm{g}_{\mathrm{j}}(\mathrm{x}) \text { for all } \mathrm{j} \neq \mathrm{i}
$$

$\mathrm{g}_{\mathrm{i}}(\mathrm{x})=\ln \mathrm{p}\left(\mathrm{w}_{\mathrm{i}}\right)-0.5 \ln \left|\sum_{\mathrm{i}}\right|-0.5\left(\mathrm{x}-\mathrm{m}_{\mathrm{i}}\right)^{\mathrm{T}} \sum_{\mathrm{i}}\left(\mathrm{x}-\mathrm{m}_{\mathrm{i}}\right)$

where 


$\begin{array}{ll}\mathrm{x} & \text { pixel vector } \\ \mathrm{w}_{\mathrm{i}} & \text { spectral class } \mathrm{i} \\ \mathrm{m}_{\mathrm{i}} \text { and } \sum \mathrm{i} & \text { mean vector and covariance }\end{array}$

matrix of the data in class $\mathrm{w}_{\mathrm{i}}$

In most cases, we assume that every class in an image has the same prior probability $\mathrm{p}\left(\mathrm{w}_{\mathrm{i}}\right)$. The image $\mathrm{R}$ is segmented successively into smaller and smaller quadrant regions so that, for any region $R_{i}, Q\left(R_{i}\right)=T R U E$. If $Q\left(R_{i}\right)=F A L S E$, the image is divided into quadrants. If $\mathrm{Q}$ is false for any quadrant, the quadrant is subdivided into subquadrants as shown in Fig 2, and so on. This particular technique has a convenient representation in the form of so-called quadtrees, that is, trees[10] in which each node has exactly four descendants as in Fig 3. The regions are termed as quadregions or quadimages. The root of the tree corresponds to the entire image and that each node corresponds to the subdivision of a node into four descendant nodes.

\begin{tabular}{|l|l|l|}
\hline $\mathrm{R}_{1}$ & \multicolumn{2}{l|}{} \\
\hline $\mathrm{R}_{3}$ & \multicolumn{2}{l|}{} \\
& $\mathrm{R}_{41}$ & $\mathrm{R}_{42}$ \\
\cline { 2 - 3 } & $\mathrm{R}_{43}$ & $\mathrm{R}_{44}$ \\
\hline
\end{tabular}

FIG 2. PARTITIONED IMAGE

If only splitting is used, the final partition normally contains adjacent regions with identical properties. To avoid this, merging is combined with splitting. Two adjacent regions $R_{i}$ and $R_{j}$ are merged only if $Q\left(R_{i} U\right.$ $\left.\mathrm{R}_{\mathrm{j}}\right)=$ TRUE.

Thus the procedure is which, at any step,

a) Split into four disjoint quadrants any region $R_{i}$ for which $\mathrm{Q}\left(\mathrm{R}_{\mathrm{i}}\right)=$ FALSE

b) When no further splitting is possible, merge any adjacent regions $R_{i}$ and $R_{j}$ for which $Q\left(R_{i} U\right.$ $\left.\mathrm{R}_{\mathrm{j}}\right)=$ TRUE

c) Stop when no further merging is possible

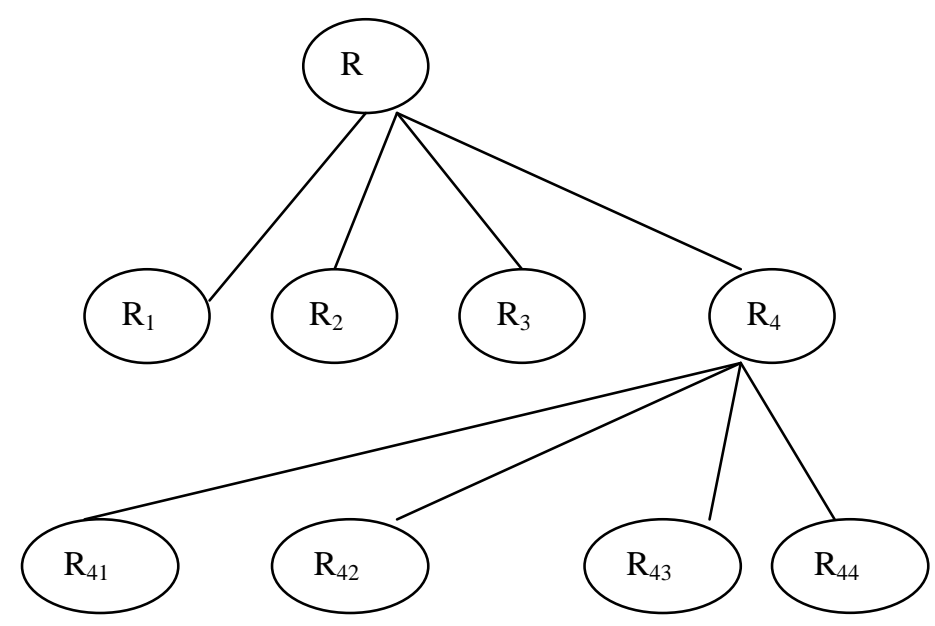

FIG 3. QUADTREE REPRESENTATION

It is customary to specify a minimum quadregion size beyond which no further splitting is carried out. The procedure is simplified by allowing any two adjacent regions $R_{i}$ and $R_{i}$ to merge if each one satisfies the predicate individually. This results in a much simpler and faster algorithm and still capable of yielding good segmentation results.

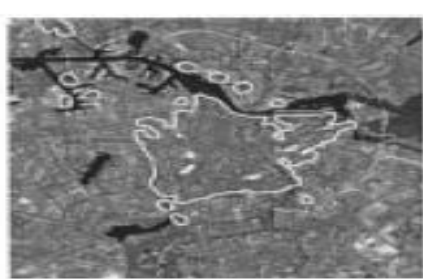

(a)

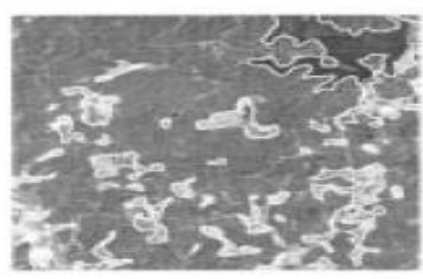

(c)

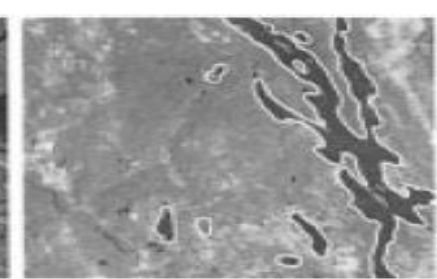

(b)

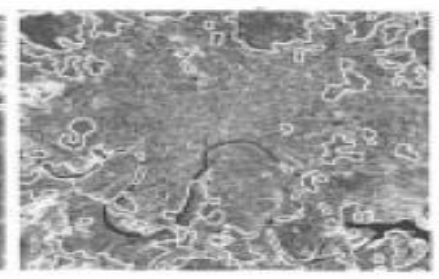

(d)

\section{FIG 4. SOME REGIONS OF INTEREST IN LANDSAT TM IMAGES}

For multispectral images, as shown in Fig 4, we can identify classes which are both data-type classes and classes of semantics. For example, one expects that textured regions and smooth regions are best compressed by different algorithms. Classes like "textured" or "smooth" are data-type classes. Classes of semantics are classes like "agricultural," "urban," etc. The classes of 
semantics are often associated with a specific data-type class.

A new compression scheme that is different from traditional image codecs is proposed. First, it extracts from the image the useful regions, corresponding to the specific application. Then it selects an appropriate compression algorithm to encode the regions thereby incorporating the advantages of different algorithms.

\section{KARHUNEN-LOEVE TRANSFORM [KLT]}

For the encoding to be efficient, the transformation phase has to be accomplished well. Since the proposed work mainly focuses on multispectral images, not only spatial but also the spectral dependencies have to be removed. The KLT is highly data dependent and hence more suitable for multispectral images. The section gives the mathematical framework of KLT followed by quadtree and class based KLT.

\section{A Mathematical Framework}

The following section describes briefly about the Karhunen-Loeve transform[14]. It is also termed as hotelling transform. The KLT minimizes the mean square error in any input image and any number of retained coefficients [13]. It is a diagonalization matrix for the auto covariance matrix of the signal. Let us denote the signal vector of ' $\mathrm{n}$ ' data points by $\mathrm{x}$ ( $\mathrm{n} \mathrm{x}$ 1matrix).

The auto covariance matrix $C_{X}$ of the vector population is defined as

$$
C_{X}=E\left[(x-\mu)(x-\mu)^{T}\right]
$$

where $\mu=E[x]$. Without loss of generality, we can assume $\mu=0$. The auto covariance matrix $C_{X}$ then becomes

$$
\mathrm{C}_{\mathrm{X}}=\mathrm{E}\left[\mathrm{xx}^{\mathrm{T}}\right]
$$

Note that $\mathrm{C}_{\mathrm{X}}$ is real and symmetric matrix. Thus, $\mathrm{C}_{\mathrm{X}}$ can be diagonalized. In other words, there exists a orthonormal matrix $M$ such that

$$
M^{T} C_{X} M=D
$$

where $\mathrm{D}$ is a diagonal matrix corresponding to the eigen values of the matrix $C_{x}$. Note that each column of $M$ is an eigenvector of $\mathrm{C}_{\mathrm{X}}$.

The KLT is defined as

$$
y=M^{T} x
$$

Now the transformed vector $\mathrm{y}$ is decorrelated ie., the spectral dependencies are removed. From (3), we have

$E\left[y^{T}\right]=E\left[M^{T} x x^{T} M\right]=M^{T} E\left[x^{T}\right] M=M^{T} C_{x} M=D(5)$

The mean square error between $\mathrm{x}$ and $\mathrm{y}$ is given as the difference between the eigen values of $\mathrm{Cx}$ and $\mathrm{Cy}$, where $\mathrm{Cy}$ is a diagonal matrix whose elements along the main diagonal are the eigen values of $\mathrm{Cx}$. The off-diagonal elements of the covariance matrix, $\mathrm{Cy}$, are 0 , so the elements of y vectors are uncorrelated.

The KLT is applied only to the 1-D spectral domain in the imagery whereas the WT is applied to the 2D spatial domain as shown in Fig. 5.

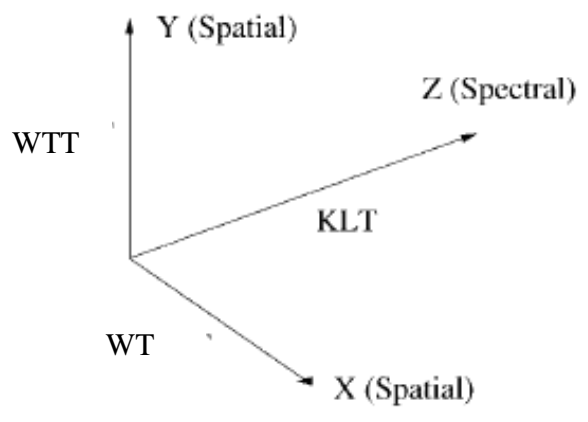

FIG 5. KLT FOR THE SPECTRAL DOMAIN AND WT FOR THE SPATIAL DOMAIN

The KLT is optimal in the sense that it minimizes the mean square error between the vectors $\mathrm{x}$ and their approximations $y$. The error can be minimized by selecting the eigen vectors associated with the largest eigen values. Thus the hotelling transform or KLT is also known as the principal components transform.B Quadtree based KLT 
Our main focus is on multispectral image, which includes smooth regions, uniformly textured regions ie., regions with same spectral characteristics [8], mixed regions etc. If a common block size is used, it seems to be inefficient due to the non uniform spectral characteristics of multispectral image. Hence, adaptive block size [14] can be used based on the regions identified.

The optimum block size is determined on the basis of Rate Distortion curve. If the block size is not limited to any threshold value, then there comes the problem in representing the block size. Hence quadtree based approach [14] proposed by J.Lee et al., is chosen. The block size depends on the quadtree. The size of quadtree depends on the number of bits used for KLT matrices and on the transform coefficient bits. Thus quadtree based KLT determines the block size based on the regions identified which in turn helps in efficient encoding.

\section{Class adaptive KLT}

The research work mainly deals with encoding the segmented regions based on the classes identified. The hotelling transform is identified as the best for region based approach [24]. The selection of KLT matrix plays the key role in transformation. A single KLT matrix can be used for all the regions ie., for the whole image. Since the work deals with classes, the spectral dependencies in the multispectral image can be efficiently removed by applying different KLT matrices [4] based on the data type ie., whether smooth or textured region. The technique is termed as class adaptive KLT.

\section{SHAPE ADAPTIVE WAVELET TRANSFORM}

Wavelet transform is easier to compress, transmit and analyze many images. In case of multispectral images, it includes regions of varying size as well as regions of arbitrary shapes. The Shape Adaptive Wavelet Transform (SAWT) suits better than conventional rectangular based wavelet transform [17]. The hotelling transform is applied to decorrelate the spectral dependency whereas SAWT is applied in two dimensions to remove the spatial dependency $[24,26]$ in the spatial domain. Unlike the block based discrete cosine transform [3], it deals with the arbitrary shapes in multispectral images efficiently.

Shape adaptive wavelet transform works as ordinary wavelet transform if the shape is rectangular in nature [16]. In an multispectral image, the regions may be large, small or even isolated points. SAWT performs the transformation of each and every region efficiently so that while decoding the image, it results in best quality image. Thus if the segmentation is done well, shape adaptive wavelet transform results in better transformation.

Better segmentation results in clearer region identification and better the regions identified, the shape adaptive wavelet transform works well. If the transformation is done efficiently, it results in better encoded image.

\section{ADAPTIVE ENCODING ALGORITHM}

Maria Petrou et al. [20] explored various compression algorithms like JPEG, SPIHT, object based wavelet method etc. Each algorithm has its own significance on different types of regions. Only semantic classes are concentrated. According to the application, particular region of interest is identified and compression is applied to the identified region[7]. The other regions are considered as unwanted portion. The compression technique is selected according to the application ie., if the application is to identify river, only the river portion is segmented and compressed using SPIHT algorithm $[2,9,18]$. The remaining portions are neglected. In the proposed work, all regions are considered as regions of interest.

The different compression algorithms thereby have their own key features [21] in terms of both visual quality and PSNR values according to the regions applied. Thus the proposed work as shown in Fig 6, is to incorporate the advantages of the algorithms in the compression of a single image, since smooth and textured regions are present in the same image and all the regions are considered as regions of interest in a Multispectral image.

Multispectral image

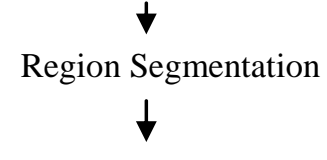

Class based KLT

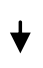

Shape adaptive WT

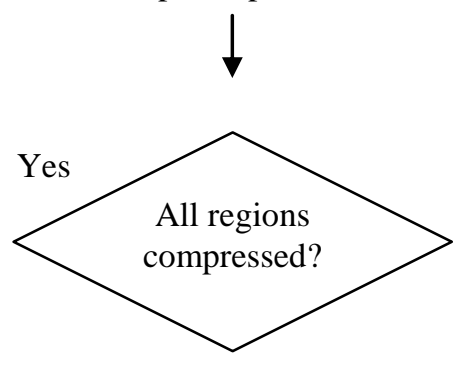




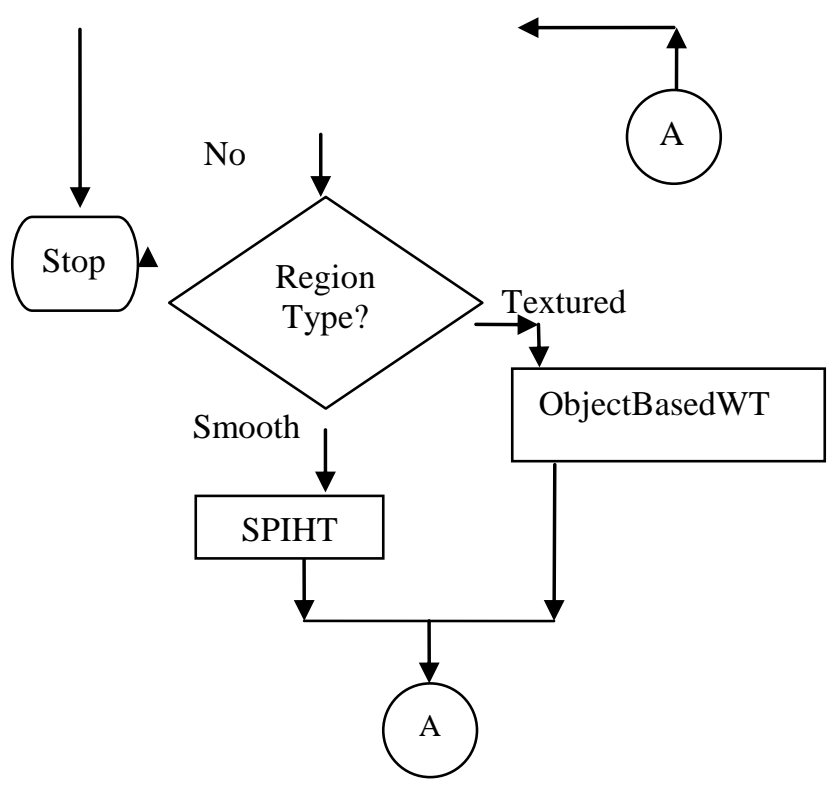

FIG 6. ADAPTIVE ENCODING ALGORITHM

The proposed algorithm can be described as follows.

1) The multispectral image is classified by segmentation. The classification is based on the predicate chosen and the regions are classified by the method 'region growing and splitting'

2) Transformation is applied to the segmented image. Class adaptive KL Transform is applied in the spectral domain and Shape adaptive Wavelet transform is applied in the spatial domain. The transformation decorrelates the spectral as well spatial dependencies.

\section{3) Repeat for each region}

i) Check the type of region by means of a statistical property, say, for example, standard deviation.

ii) If a region is smooth, the SPIHT encoding technique is applied.

Else

If a region is highly textured, the object based wavelet encoding method is applied for compression.

Until (all regions are explored)

\section{CONCLUSION AND FUTURE WORK}

The proposed work is a region based adaptive scheme for multispectral image compression. The image is first segmented into many regions by region splitting and merging technique. Then it follows transformation in both spectral and spatial domain. It considers the spectral and statistical properties which are unique to multispectral images. The class adaptive KL transform adopted in the spectral domain uses a single KLT matrix for the regions of same class thereby brings advantages in terms of computation cost and side information. It also considers the statistical properties of each class. The shape adaptive Wavelet transform is robust and works well even in very small regions. The encoding algorithm adopts two techniques based on data type classes, ie., smooth or textured region.

The region based coder is the most appropriate scheme, in the sense, it incorporates the advantages of both SPIHT algorithm and object based wavelet method in a single compression technique. It is particularly suitable for multispectral images where smooth and textured regions are common in a single image. In future research, an RDdriven segmentation technique, with a split-and-merge procedure where each step is guided by the actual RD performance gain/loss measured on the image to be coded can be used. Segmentation should consider the problem of mixed cells so that even smaller regions are classified efficiently which plays a key role in adaptive coding algorithm.

\section{REFERENCES}

[1] A. Gersho and R. M. Gray, Vector Quantization and Signal Compression. Boston, MA: Kluwer, 1992.

[2] A. Said and W. A. Pearlman, "A new fast and efficient image codec based on set partitioning in hierarchical trees," IEEE Trans. Circuits Syst. Video Technol., vol. 6, no. 6, pp. 243-250, Jun. 1996.

[3] B. Kim and W. A. Pearlman, "An embedded wavelet video coder using three-dimensional set partitioning in hierarchical trees," in Proc. Data Compression Conf., Mar. 1997, pp. 251260.

[4] B. Penna, T. Tillo, E. Magli, and G. Olmo, "A new low complexity KLT for lossy hyperspectral data compression," in Proc. IEEE Int. Geoscience and Remote Sensing Symp., Aug. 2006, pp. 3528-3528.

[5] B. Penna, T. Tillo, E. Magli, and G. Olmo, "Transform coding techniques for lossy hyperspectral data compression," IEEE Geosci. Remote Sens. Lett., vol. 45, no. 5, pp. 1408-1421, May 2007. 
[6] C. D’Elia, G. Poggi, and G. Scarpa, “A tree-structured markov random field model for bayesian image segmentation," IEEE Trans. Image Process., vol. 12, no. 10, pp. 1259-1273, Oct. 2003.

[7] G. Fernandez and C. M. Wittenbrink, "Coding of spectrally homogeneous regions in multispectral image compression," in Proc. IEEE Int. Conf. Image Processing, Lausanne, Switzerland, Sep. 1996, vol. 2, pp. 923-926.

[8] G. Gelli and G. Poggi, "Compression of multispectral images by spectral classification and transform coding," IEEE Trans. Image Process., vol. 8, no. 4, pp. 476-489, Apr. 1999.

[9] G. P. Abousleman, M. W. Marcellin, and B. R. Hunt, "Compression of hyperspectral imagery using the 3-D DCT and hybrid DPCM/DCT," IEEE Trans. Geosci. Remote Sens., vol. 33, no. 1, pp. 26-34, Jan. 1995.

[10] G. Poggi and A. R. P. Ragozini, "Image segmentation by tree-structured Markov random fields," IEEE Signal Process. Lett., vol. 6, no. 7, pp. 155-157, Jul. 1999.

[11] G. R. Canta and G. Poggi, "Kronecker-product gain-shape vector quantization for multispectral and hyperspectral image coding," IEEE Trans. Image Process., vol. 7, no. 5, pp. 668-678, May 1998.

[12] J. A. Saghri, A. G. Tescher, and J. T. Reagan, "Practical transform coding of multispectral imagery," IEEE Signal Process. Mag., vol. 12, no. 1, pp. 32-43, Jan. 1995.

[13] J. E. Fowler and D. N. Fox, "Embedded wavelet-based coding of threedimensional oceanographic images with land masses," IEEE Trans. Geosci. Remote Sens., vol. 39, no. 1, pp. 284-290, Feb. 2001.

[14] J. Lee, "Optimized quadtree for Karhunen-Loève transform in multispectral image coding," IEEE Trans. Image Process., vol. 8, no. 4, pp.453-461, Apr. 1999.

[15] Marco Cagnazzo, Giovanni Poggi, and Luisa Verdoliva, "Region-Based Transform Coding of Multispectral Images", IEEE Transactions On Image Processing, Vol. 16, No. 12, Pg. 2916-2927, December 2007

[16] M. Cagnazzo, S. Parrilli, G. Poggi, and L. Verdoliva, "Improved class-based coding of multispectral images with shape- adaptive wavelet transform," IEEE Geosci. Remote Sens. Lett., vol. 4, no. 4, pp. 566-570, Oct. 2007.

[17] M. Cagnazzo, S. Parrilli, G. Poggi, and L. Verdoliva, "Costs and advantages of object-based image coding with shape-adaptive wavelet transform," Int. J. Image Video Process., vol. 2007, p. 13, 2007, Article ID 78323.

[18] M. Cagnazzo, G. Poggi, L. Verdoliva, and A. Zinicola, "Region-oriented compression of multispectral images by shapeadaptive wavelet transform and SPIHT," in Proc. IEEE Int. Conf. Image Processing, Singapore, Oct. 2004, pp. 2459-2462.

[19] M. Finelli, G. Gelli, and G. Poggi, "Multispectral image coding by spectral classification," in Proc. IEEE Int. Conf. Image Process., Lausanne, Switzerland, Sep. 1996, vol. 2, pp. 605-608.

[20] M. Petrou, P. Hou, S. Kamata, and C. I. Underwood, "Region-based image coding with multiple algorithms," IEEE Trans. Geosci. Remote Sens., vol. 41, no. 3, pp. 562-570, Mar. 2001.

[21] Nikola Sprljan, Sonja Grgic, Mislav Grgic, "Modified SPIHT algorithm for wavelet packet image coding" Real-Time Imaging 11 (2005) 378-388, www.sciencedirect.com

[22] P. L. Dragotti, G. Poggi, and A. R. P. Ragozini, "Compression of multispectral images by three-dimensional SPIHT algorithm," IEEE Trans. Geosci. Remote Sens., vol. 38, no. 1, pp. 416-428, Jan. 2000.

[23]Q. Du and J. E. Fowler, "Hyperspectral image compression using JPEG2000 and principal component analysis," IEEE Geosci. Remote Sens. Lett., vol. 45, no. 4, pp. 201-205, Apr. 2007.

[24] S. E. Qian, "Hyperspectral data compression using a fast vector quantization algorithm," IEEE Trans. Geosci. Remote Sens., vol. 42, no. pp. 1791-1798, Aug. 2004.

[25] S. Gupta and A. Gersho, "Feature predictive vector quantization multispectral images," IEEE Geosci. Remote Sens. Lett., vol. 30, no. 5, pp. 491-501, May 1992.

[26] S. Li and W. Li, "Shape-adaptive discrete wavelet transforms for arbitrarily shaped visual object coding," IEEE Trans. Circuits Syst. Video Technol., vol. 10, no. 8, pp. 725-743, Aug. 2000. 